\title{
Limits on Lorentz invariance violation at the Planck energy scale from H.E.S.S. spectral analysis of the blazar Mrk 501
}

\author{
Matthias Lorentz ${ }^{1, a}$ and Pierre Brun ${ }^{1, b}$, for the H.E.S.S. collaboration \\ ${ }^{1}$ Irfu, CEA Saclay, 91191 Gif-sur-Yvette France
}

\begin{abstract}
Some extensions to the Standard Model lead to the introduction of Lorentz symmetry breaking terms, expected to induce deviations from Lorentz symmetry around the Planck scale. A parameterization of effects due to Lorentz invariance violation (LIV) can be introduced by adding an effective term to the photon dispersion relation. This affects the kinematics of electron-positron pair creation by $\mathrm{TeV} \gamma$ rays on the extragalactic background light (EBL) and translates into modifications of the standard EBL opacity for the $\mathrm{TeV}$ photon spectra of extragalactic sources. Exclusion limits are presented, obtained with the spectral analysis of H.E.S.S. observations taken on the blazar Mrk 501 during the exceptional 2014 flare. The energy spectrum, extending very significantly above 10 $\mathrm{TeV}$, allows to place strong limits on LIV in the photon sector at the level of the Planck energy scale for linear perturbations in the photon dispersion relation, and provides the strongest constraints presently for the case of quadratic perturbations.
\end{abstract}

\section{Introduction}

Special relativity is a pillar of modern physics and Lorentz symmetry has been established to be an exact symmetry of Nature up to the precision of current experiments. It has been suggested however that this symmetry could only be approximate and that deviations from Lorentz invariance could appear at an energy scale beyond our current grasp. A generic approach to introduce such effects consists of adding effective terms in the dispersion relation of particles, i.e. for photons

$$
E_{\gamma}^{2}=p_{\gamma}^{2} \pm E_{\gamma}^{2}\left(\frac{E_{\gamma}}{E_{\mathrm{LIV}}}\right)^{n}
$$

where $E_{\mathrm{LIV}}$ is the hypothetical energy scale at which Lorentz symmetry could stop being exact, and $n$ the order of the leading correction. In some approaches to quantum gravity $E_{\text {LIV }}$ is expected to be of the order of Planck energy $\mathrm{E}_{\text {Planck }}=\sqrt{\hbar c^{5} / G} \simeq 1.22 \times 10^{28} \mathrm{eV}$ [3]. As such deviations are only expected for photons at the highest energies, astrophysical $\gamma$-rays can be used to probe potential LIV effects. The most widely-used approach is to look for energy-dependent time delays for photons produced by distant $\gamma$-ray bursts (GRB) or during TeV flares of active galaxy nuclei (AGN), see e.g. [21]. An attractive alternative possibility takes advantage of the fact that the modified dispersion relation for photons that could be induced by LIV would affect the kinematics for the $e^{+} e^{-}$pair production of

\footnotetext{
a e-mail: matthias.lorentz@cea.fr

be-mail: pierre.brun@cea.fr
} 
$\mathrm{TeV} \gamma$ rays coming from AGNs on the EBL resulting in a modified opacity to extragalactic $\gamma$ rays, see $e . g$. [11]. In the following we consider LIV affecting only photons (like in $[12,13]$ ), not electrons as the constraints on LIV for electrons are very stringent due to observations of synchrotron radiation from the Crab Nebula [18].

\section{Modified EBL opacity in the presence of LIV}

The EBL is the background photon field originating from the integrated starlight and its reprocessing by the interstellar medium over cosmic history. Its spectral energy distribution has two main components, an optical $(\sim 1 \mathrm{eV})$ and an infrared $\left(\sim 10^{-2} \mathrm{eV}\right)$ component. Extragalactic very high-energy (VHE, E $>100 \mathrm{GeV}$ ) $\gamma$ rays can be used as an independent way to probe this background radiation, as such $\gamma$ rays interact with EBL photons via $e^{+} e^{-}$pair production [1], resulting in an attenuated observed flux (for a review see [2]). The optical depth for a VHE photon of energy $E_{\gamma}$ traveling through a medium with EBL physical density $n(\epsilon, z)$ from a source at $z_{s}$ is:

$$
\tau\left(E_{\gamma}, z_{s}\right)=c \int_{0}^{z_{s}} d z \frac{d t}{d z} \int_{0}^{2} d \mu \frac{\mu}{2} \int_{\epsilon_{\text {thr }}}^{\infty} d \epsilon \frac{d n(\epsilon, z)}{d \epsilon} \sigma_{\gamma \gamma}\left(E_{\gamma}(1+z), \epsilon, \mu\right),
$$

where $d t / d z=\left(H_{0}(1+z) \sqrt{\Omega_{\mathrm{M}}(1+z)^{3}+\Omega_{\Lambda}}\right)^{-1} 1, \mu=1-\cos (\theta), \epsilon_{t h r}\left(E_{\gamma}, z\right)=\frac{2 m_{e}^{2} c^{4}}{E_{\gamma} \mu(1+z)}$ and $\sigma_{\gamma \gamma}$ is the Bethe-Heitler cross section for pair production. The absorption effect on the intrinsic spectrum of an extragalactic source is expressed as $\Phi_{\text {obs }}\left(E_{\gamma}\right)=\Phi_{\text {int }}\left(E_{\gamma}\right) \mathrm{e}^{-\tau\left(E_{\gamma}, z_{s}\right)}$. EBL absorption then leaves a typical redshift and energy-dependent imprint on the observed spectrum of extragalactic sources. Knowledge of the EBL spectral energy distribution has greatly improved over the last decade, constraints from VHE $\gamma$ rays (see e.g. [6-8]), predictions from models (see e.g. [4, 5]), and results from an empirical determination [9] agree in between lower and upper limits.

The effective dispersion relation in the presence of LIV Eq.1 propagates into the optical depth given in Eq.2, the invariant center-of-mass energy squared $s$ and threshold energy $\epsilon_{\text {thr }}$ become :

$$
s \rightarrow s \pm \frac{E_{\gamma}^{n+2}}{E_{\mathrm{LIV}}^{n}}, \text { and } \epsilon_{\mathrm{thr}} \rightarrow \epsilon_{\mathrm{thr}} \mp \frac{1}{4} \frac{E_{\gamma}^{n+1}}{E_{\mathrm{LIV}}^{n}}
$$

We assume, as in [12], that the modified expression of $s$ can still be considered as an invariant quantity in the LIV framework (for a discussion see Appendix A. in [13]). We only consider the subluminal case (minus sign in Eq. 1) : if non negligible, the effective term will induce lower values for $s$ suppressing pair creation on the EBL, causing an excess of transparency for $\gamma$ rays $^{2}$, see Fig. 1 .

\section{H.E.S.S. observations of Mrk 501 during the 2014 flare}

\subsection{H.E.S.S. experiment}

The High Energy Stereoscopic System (H.E.S.S.) is an array of five imaging atmospheric Cherenkov telescopes located $1800 \mathrm{~m}$ above see level in the Khomas Highland, Namibia, detecting $\gamma$-rays ranging from $\sim 100 \mathrm{GeV}$ to a few tens of $\mathrm{TeV}$.

\footnotetext{
${ }^{1}$ We assume a flat $\Lambda C D M$ cosmology with $\Omega_{\mathrm{M}}=0.3, \Omega_{\Lambda}=0.7$ and $H_{0}=70 \mathrm{~km} \mathrm{~s}^{-1} \mathrm{Mpc}^{-1}$.

${ }^{2}$ Alternatively, in the superluminal case the threshold energy would be lowered implying an enhanced pair production for $\gamma$ rays that could more easily interact with the cosmic microwave background. This would result in a strong cut-off in the observed energy spectra of AGNs. This LIV scenario is unlikely with respect to current observations and also theoretically disfavored.
} 


\subsection{Mrk 501}

Mrk 501 is a well known AGN at redshift $z=0.034$ which belongs to the class of blazars, i.e. with its relativistic jet closely aligned to our line of sight. It is known to be highly variable from radio to VHE $\gamma$ rays and is referred to as a high-frequency-peaked blazar with a flux-dependent spectral hardening observed during flaring states. Its spectral characteristics and its relatively low redshift allow for the detection of among the most energetic extragalactic $\gamma$ rays, making this source ideal to investigate LIV through spectral studies as it has already been done [7, 10, 13] with the historically highest VHE flux recorded in 1997 by the HEGRA [17] and CAT [16] telescopes.

\subsection{Flare data set}

The 2014 H.E.S.S. observations of Mrk 501 were triggered following high fluxes reported by the FACT collaboration. Observations taken during the night of June 23-24 2014 (MJD 56831-56832) revealed an exceptional flare with highest fluxes of Mrk 501 ever recorded by H.E.S.S. [14]. These observations were performed with full array of all five telescopes, however for this study requiring optimal sensitivity at highest energies, data from the central large telescope are not essential. The mean zenith angle of observations was $\sim 63^{\circ}$. The Model analysis with loose cuts [19] was performed leading to an excess of more than 1200 photons with a $\sim 67 \sigma$ significance for the 2 hours of observations taken that night. Spectral analysis was performed using the forward folding technique described in [20]. The spectrum, extending significantly up to $\sim 20 \mathrm{TeV}$, is well fitted $\left(\chi^{2} /\right.$ n.d.f $\left.=8.5 / 8\right)$ by a simple EBL-absorbed power law using the EBL model of [4]. There is no evidence for intrinsic curvature nor cut-off. The fitted intrinsic index is $2.15 \pm 0.06$ [15].

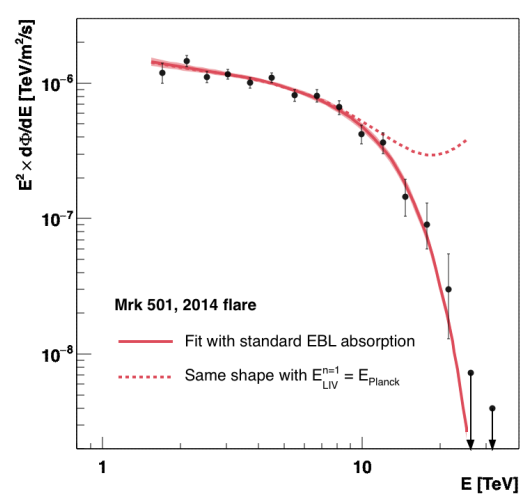

Figure 1. Energy spectrum of Mrk 501 obtained from the H.E.S.S. phase-I analysis of the 2014 flare data. The fitted EBL-absorbed power law for the standard case is showed by the solid line, as well with the corresponding $1-\sigma$ confidence band. For comparison the same intrinsic power law with modified EBL absorption due to linear Planck scale perturbations is represented by the dashed line.

\section{Results and discussion}

The maximum likelihood forward folding method for spectrum determination is performed assuming an intrinsic power law absorbed with the EBL model of [4]. The optical depths are computed considering modifications due to LIV as explained in Sec. 2. Values of $E_{\mathrm{LIV}}$ are scanned logarithmically in the range of interest for linear $(n=1)$ and quadratic $(n=2)$ scenarios. As the data show no evidence for a high-energy upturn, the fit prefers LIV-free optical depth values. Indeed log-likelihood 
values reach plateaus corresponding to the standard case with no deviations from Lorentz symmetry in both cases. This allows to compute exclusion limits on $E_{\mathrm{LIV}}$, as summarized in Tab. 1 .

Table 1. Exclusion limits obtained on $E_{\mathrm{LIV}}$ at different confidence levels.

\begin{tabular}{|l|l|l|l|}
\hline & \multicolumn{1}{|c|}{$2 \sigma$} & \multicolumn{1}{|c|}{$3 \sigma$} & \multicolumn{1}{c|}{$5 \sigma$} \\
\hline $\mathrm{n}=1$ & $2.8 \times 10^{28} \mathrm{eV}\left(2.29 \times \mathrm{E}_{\text {Planck }}\right)$ & $1.9 \times 10^{28} \mathrm{eV}\left(1.6 \times \mathrm{E}_{\text {Planck }}\right)$ & $1.04 \times 10^{28} \mathrm{eV}\left(0.86 \times \mathrm{E}_{\text {Planck }}\right)$ \\
\hline $\mathrm{n}=2$ & $7.5 \times 10^{20} \mathrm{eV}$ & $6.4 \times 10^{20} \mathrm{eV}$ & $4.7 \times 10^{20} \mathrm{eV}$ \\
\hline
\end{tabular}

These strong constraints naturally come from the exceptional spectrum of the 2014 flare data-set where the power law intrinsic emission extends up to $20 \mathrm{TeV}$. A cross-check analysis using independent calibration and reconstruction chains lead to a compatible spectrum. The same LIV analysis using the EBL model of [5] leads to very similar exclusion limits.

\section{Conclusions}

The non observation of deviations from standard EBL absorption in the multi-TeV spectrum of Mrk 501 observed by H.E.S.S. during the 2014 flare allows us to derive strong limits on $E_{\mathrm{LIV}}$ in the photon sector, currently the best limits obtained with an AGN. This confirms the result obtained with GRB 090510 [22] that standard photon dispersion relation holds up to the Planck energy scale in the case of linear perturbations, and pushes higher the current limit in the case of quadratic perturbations.

\section{Acknowledgments}

Please see standard acknowledgements in H.E.S.S. papers, not reproduced here due to lack of space.

\section{References}

[1] Gould, R. J., \& Schréder, G. P. 1967, Physical Review, 155, 1404

[2] Dwek, E., \& Krennrich, F. 2013, Astroparticle Physics, 43, 112

[3] G. Amelino-Camelia, Living Rev. Rel. 16 (2013) 5 [arXiv:0806.0339 [gr-qc]].

[4] Franceschini, A., Rodighiero, G., \& Vaccari, M. 2008, A\&A, 487, 837

[5] Dominguez, A., Primack, J. R., Rosario, D. J., et al. 2011, MNRAS, 410, 2556

[6] H.E.S.S. Collaboration, Abramowski, A., Acero, F., et al. 2013, A\&A, 550, A4

[7] J. Biteau and D. A. Williams, Astrophys. J. 812 (2015) no.1, 60 [arXiv:1502.04166 [astro-ph.CO]].

[8] M. Lorentz et al. [HESS Collaboration], arXiv:1509.03477 [astro-ph.HE].

[9] F. W. Stecker, S. T. Scully and M. A. Malkan, arXiv:1605.01382 [astro-ph.HE].

[10] F. W. Stecker and S. L. Glashow, Astropart. Phys. 16 (2001) 97 [astro-ph/0102226].

[11] U. Jacob and T. Piran, Phys. Rev. D 78 (2008) 124010 [arXiv:0810.1318 [astro-ph]].

[12] M. Fairbairn, A. Nilsson, J. Ellis, J. Hinton and R. White, JCAP 1406 (2014) 005 [arXiv:1401.8178 [astro-ph.HE]].

[13] Tavecchio, F., \& Bonnoli, G. 2016, A\&A, 585, A25

[14] N. Chakraborty et al. [HESS Collaboration], arXiv:1509.04893 [astro-ph.HE].

[15] G. Cologna et al. [HESS Collaboration], arXiv:1509.04458 [astro-ph.HE].

[16] D A. Djannati-Atai et al., Astron. Astrophys. 350 (1999) 17 [astro-ph/9906060].

[17] F. Aharonian [HEGRA Collaboration], Astron. Astrophys. 349 (1999) 11 [astro-ph/9903386].

[18] T. Jacobson, S. Liberati and D. Mattingly, Nature 424 (2003) 1019 [astro-ph/0212190].

[19] de Naurois, M., \& Rolland, L. 2009, Astroparticle Physics, 32, 231

[20] F. Piron et al., Astron. Astrophys. 374 (2001) 895 [astro-ph/0106196].

[21] A. Abramowski et al. [HESS Collaboration], Astropart. Phys. 34 (2011) 738 [arXiv:1101.3650 [astro-ph.HE]].

[22] V. Vasileiou et al., Phys. Rev. D 87 (2013) no.12, 122001 [arXiv:1305.3463 [astro-ph.HE]]. 